\title{
Approximation of eigenvalues of boundary value problems
}

\author{
Mohammed M Tharwat ${ }^{1,2^{*}}$ and Saleh M Al-Harbi ${ }^{3}$
}

${ }^{\text {"Correspondence: }}$

zahraa26@yahoo.com

1 Department of Mathematics, Faculty of Science, King Abdulaziz

University, North Jeddah, Saudi Arabia

${ }^{2}$ Permanent address: Department of Mathematics, Faculty of Science, Beni-Suef University, Beni-Suef, Egypt

Full list of author information is available at the end of the article

\begin{abstract}
In the present paper we apply a sinc-Gaussian technique to compute approximate values of the eigenvalues of discontinuous Dirac systems, which contain an eigenvalue parameter in one boundary condition, with transmission conditions at the point of discontinuity. The error of this method decays exponentially in terms of the number of involved samples. Therefore the accuracy of the new technique is higher than the classical sinc-method. Numerical worked examples with tables and illustrative figures are given at the end of the paper showing that this method gives us better results.
\end{abstract}

MSC: 34L16; 94A20; 65L15

Keywords: sinc-Gaussian; sinc-method; Dirac systems; transmission conditions; discontinuous boundary value problems; truncation and amplitude errors

\section{Introduction}

Consider the discontinuous Dirac system which consists of the system of differential equations

$$
\left(\begin{array}{c}
y_{2}^{\prime}(x)-r_{1}(x) y_{1}(x) \\
y_{1}^{\prime}(x)+r_{2}(x) y_{2}(x)
\end{array}\right)=\left(\begin{array}{c}
\lambda y_{1}(x) \\
-\lambda y_{2}(x)
\end{array}\right), \quad x \in[-1,0) \cup(0,1],
$$

with boundary conditions

$$
\begin{aligned}
& U_{1}(\mathbf{y}):=\sin \alpha y_{1}(-1)-\cos \alpha y_{2}(-1)=0, \\
& U_{2}(\mathbf{y}):=\left(a_{1}+\lambda \sin \beta\right) y_{1}(1)-\left(a_{2}+\lambda \cos \beta\right) y_{2}(1)=0
\end{aligned}
$$

and transmission conditions

$$
\begin{aligned}
& U_{3}(\mathbf{y}):=y_{1}\left(0^{-}\right)-\delta y_{1}\left(0^{+}\right)=0, \\
& U_{4}(\mathbf{y}):=y_{2}\left(0^{-}\right)-\delta y_{2}\left(0^{+}\right)=0,
\end{aligned}
$$

where $\lambda \in \mathbb{C} ; \mathbf{y}=\left(\begin{array}{l}y_{1} \\ y_{2}\end{array}\right)$; the real-valued functions $r_{1}(\cdot)$ and $r_{2}(\cdot)$ are continuous in $[-1,0)$ and $(0,1]$, and have finite limits $r_{1}\left(0^{ \pm}\right):=\lim _{x \rightarrow 0^{ \pm}} r_{1}(x), r_{2}\left(0^{ \pm}\right):=\lim _{x \rightarrow 0^{ \pm}} r_{2}(x) ; a_{1}, a_{2}, \delta \in$ $\mathbb{R}, \alpha, \beta \in[0, \pi) ; \delta \neq 0$ and $\rho:=a_{1} \cos \beta-a_{2} \sin \beta>0$.The aim of the present work is to compute the eigenvalues of (1.1)-(1.5) numerically by the sinc-Gaussian technique with errors analysis, truncation error and amplitude error.

@2014 Tharwat and Al-Harbi; licensee Springer. This is an Open Access article distributed under the terms of the Creative Commons Attribution License (http://creativecommons.org/licenses/by/2.0), which permits unrestricted use, distribution, and reproduction in any medium, provided the original work is properly cited. 
Sampling theory is one of the most important mathematical tools used in communication engineering since it enables engineers to reconstruct signals from some of their sampled data. A fundamental result in information theory is the Whittaker-Kotel'nikovShannon (WKS) sampling theorem [1-3]. It states that any $f \in \mathcal{B}_{\sigma}^{2}, \sigma>0$,

$$
\mathcal{B}_{\sigma}^{2}:=\left\{f: f \text { entire, }|f(\mu)| \leq C e^{\sigma|\Im \mu|}, \int_{\mathbb{R}}|f(\mu)|^{2} d \mu<\infty\right\}
$$

can be reconstructed from its sampled values $\{f(n \pi / \sigma): n \in \mathbb{Z}\}$ by the formula

$$
f(\lambda)=\sum_{n \in \mathbb{Z}} f(n \pi / \sigma) \operatorname{sinc}(\sigma \lambda-n \pi), \quad \lambda \in \mathbb{C}
$$

where

$$
\operatorname{sinc}(\lambda):= \begin{cases}\frac{\sin (\lambda)}{\lambda}, & \lambda \neq 0 \\ 1, & \lambda=0\end{cases}
$$

Series (1.6) converges absolutely and uniformly on compact subsets of $\mathbb{C}$, and uniformly on $\mathbb{R}, c f$. [4]. Expansion (1.6) is used in several approximation problems which are known as sinc-methods; see, e.g., [5-8]. In particular the sinc-method is used to approximate eigenvalues of boundary value problems; see, for example, [9-12]. The sinc-method has a slow rate of decay at infinity, which is as slow as $O\left(\left|\lambda^{-1}\right|\right)$. There have been several attempts to improve the rate of decay. One of the interesting ways is to multiply the sinc-function in (1.6) by a kernel function; see, e.g., [13-15]. Let $h \in(0, \pi / \sigma]$ and $\gamma \in$ $(0, \pi-h \sigma)$. Assume that $\Phi \in \mathcal{B}_{\gamma}^{2}$ such that $\Phi(0)=1$, then for $\mathfrak{g} \in \mathcal{B}_{\sigma}^{2}$ we have the expansion [16]

$$
\mathfrak{g}(\lambda)=\sum_{n=-\infty}^{\infty} \mathfrak{g}(n h) \operatorname{sinc}\left(h^{-1} \pi \lambda-n \pi\right) \Phi\left(h^{-1} \lambda-n\right)
$$

The speed of convergence of the series in (1.8) is determined by the decay of $|\Phi(\lambda)|$. But the decay of an entire function of exponential type cannot be as fast as $e^{-c|x|}$ as $|x| \rightarrow \infty$ for some positive $c$ [16]. In [17], Qian has introduced the following regularized sampling formula. For $h \in(0, \pi / \sigma], N \in \mathbb{N}$ and $r>0$, Qian defined the operator [17]

$$
\left(G_{h, N} \mathfrak{g}\right)(\lambda)=\sum_{n \in Z_{N}(\lambda)} \mathfrak{g}(n h) \operatorname{sinc}\left(h^{-1} \pi \lambda-n \pi\right) G\left(\frac{\lambda-n h}{\sqrt{2} r h}\right), \quad \lambda \in \mathbb{R},
$$

where $G(t):=\exp \left(-t^{2}\right)$, which is called the Gaussian function, $Z_{N}(x):=\left\{n \in \mathbb{Z}: \mid\left[h^{-1} x\right]-\right.$ $n \mid \leq N\}$ and $[x]$ denotes the integer part of $x \in \mathbb{R}$; see also [18, 19]. Qian also derived the following error bound. If $\mathfrak{g} \in \mathcal{B}_{\sigma}^{2}, h \in(0, \pi / \sigma]$ and $a:=\min \{r(\pi-h \sigma),(N-2) / r\} \geq 1$, then $[17,18]$

$$
\left|\mathfrak{g}(\lambda)-\left(G_{h, N} \mathfrak{g}\right)(\lambda)\right| \leq \frac{2 \sqrt{\sigma \pi}\|\mathfrak{g}\|_{2}}{\pi^{2} a^{2}}\left(\sqrt{2 \pi} a+e^{3 / 2 r^{2}}\right) e^{-a^{2} / 2}, \quad \lambda \in \mathbb{R}
$$


In [16] Schmeisser and Stenger extended the operator (1.9) to the complex domain $\mathbb{C}$. For $\sigma>0, h \in(0, \pi / \sigma]$ and $\omega:=(\pi-h \sigma) / 2$, they defined the operator [16]

$$
\left(\mathcal{G}_{h, N} \mathfrak{g}\right)(\lambda):=\sum_{n \in \mathbb{Z}_{N}(\lambda)} \mathfrak{g}(n h) S_{n}\left(\frac{\pi \lambda}{h}\right) G\left(\frac{\sqrt{\omega}(\lambda-n h)}{\sqrt{N} h}\right)
$$

where $\mathbb{Z}_{N}(\lambda):=\left\{n \in \mathbb{Z}:\left|\left[h^{-1} \Re \lambda+1 / 2\right]-n\right| \leq N\right\}$ and $N \in \mathbb{N}$. Note that the summation limits in (1.11) depend on the real part of $\lambda$. Schmeisser and Stenger [16] proved that if $\mathfrak{g}$ is an entire function such that

$$
|\mathfrak{g}(\xi+i \eta)| \leq \phi(|\xi|) e^{\sigma|\eta|}, \quad \xi, \eta \in \mathbb{R}
$$

where $\phi$ is a non-decreasing, non-negative function on $[0, \infty)$ and $\sigma \geq 0$, then for $h \in(0, \pi / \sigma), \omega:=(\pi-h \sigma) / 2, N \in \mathbb{N},|\Im \lambda|<N$, we have

$$
\begin{aligned}
& \left|\mathfrak{g}(\lambda)-\left(\mathcal{G}_{h, N} \mathfrak{g}\right)(\lambda)\right| \\
& \quad \leq 2\left|\sin \left(h^{-1} \pi \lambda\right)\right| \phi(|\Re \lambda|+h(N+1)) \frac{e^{-\omega N}}{\sqrt{\pi \omega N}} \beta_{N}\left(h^{-1} \Im \lambda\right), \quad \lambda \in \mathbb{C},
\end{aligned}
$$

where

$$
\beta_{N}(t):=\cosh (2 \omega t)+\frac{2 e^{\omega t^{2} / N}}{\sqrt{\pi \omega N}\left[1-(t / N)^{2}\right]}+\frac{1}{2}\left[\frac{e^{2 \omega t}}{e^{2 \pi(N-t)}-1}+\frac{e^{-2 \omega t}}{e^{2 \pi(N+t)}-1}\right] .
$$

The amplitude error arises when the exact values $\mathfrak{g}(n h)$ of (1.11) are replaced by the approximations $\tilde{\mathfrak{g}}(n h)$. We assume that $\tilde{\mathfrak{g}}(n h)$ are close to $\mathfrak{g}(n h)$, i.e., there is $\varepsilon>0$ sufficiently small such that

$$
\sup _{n \in \mathbb{Z}_{n}(\lambda)}|\mathfrak{g}(n h)-\widetilde{\mathfrak{g}}(n h)|<\varepsilon
$$

Let $h \in(0, \pi / \sigma), \omega:=(\pi-h \sigma) / 2$ and $N \in \mathbb{N}$ be fixed numbers. The authors in [20] proved that if (1.15) is held, then for $|\Im \lambda|<N$, we have

$$
\left|\left(\mathcal{G}_{h, N} \mathfrak{g}\right)(\lambda)-\left(\mathcal{G}_{h, N} \widetilde{\mathfrak{g}}\right)(\lambda)\right| \leq A_{\varepsilon, N}(\Im \lambda)
$$

where

$$
A_{\varepsilon, N}(\Im \lambda)=2 \varepsilon e^{-\omega / 4 N}(1+\sqrt{N / \omega \pi}) \exp \left((\omega+\pi) h^{-1}|\Im \lambda|\right) .
$$

Without eigenparameter appearing in any of boundary conditions, in [21] and [12] Tharwat et al. approximately computed the eigenvalues of the discontinuous Dirac system which is studied in the monographs of [22] by Hermite interpolations and regularized sinc-methods, respectively. In the regularized sinc-method, also the same in the Hermite interpolations method, the basic idea is as follows: The eigenvalues are characterized as the zeros of an analytic function $F(\lambda)$ which can be written in the form $F(\lambda)=f_{0}(\lambda)+f(\lambda)$, where $f_{0}(\lambda)$ is a known part. The ingenuity of the approach is in trying to choose the 
function $F(\lambda)$ so that $f(\lambda) \in \mathcal{B}_{\sigma}^{2}$ (unknown part) and can be approximated by the WKS sampling theorem if its values at some equally spaced points are known; see [9-12]. Recall that, in regularized sinc and Hermite interpolations methods, it is necessary that $f(\lambda)$ is an $L^{2}$-function. In this paper we will use the sinc-Gaussian sampling formula (1.11) to compute eigenvalues of (1.1)-(1.5) numerically. As is expected, the new method reduced the error bounds remarkably (see the examples in Section 4). Also here, the basic idea is to write the function of eigenvalues as the sum of two terms, one known and the other unknown but an entire function of exponential type which satisfies (1.12). In other words, the unknown term is not necessarily an $L^{2}$-function. Then we approximate the unknown part using (1.11) and obtain better results. We would like to mention that the papers in computing eigenvalues by the sinc-Gaussian method are few; see [20, 23-25]. In Sections 2, 3 we derive the sinc-Gaussian technique to compute the eigenvalues of (1.1)-(1.5) with error estimates. The last section involves some illustrative examples.

\section{Preliminaries}

In this section we derive approximate values of the eigenvalues of problem (1.1)-(1.5). Recall that problem (1.1)-(1.5) has a denumerable set of real and simple eigenvalues, $c f$. [26]; see also [22, 27-29]. Let

$$
\mathfrak{y}(\cdot, \lambda)=\left(\begin{array}{l}
\mathfrak{y}_{1}(\cdot, \lambda) \\
\mathfrak{y}_{2}(\cdot, \lambda)
\end{array}\right), \quad \mathfrak{y}_{i}(x, \lambda)= \begin{cases}\mathfrak{y}_{i 1}(x, \lambda), & x \in[-1,0), \quad i=1,2 \\
\mathfrak{y}_{i 2}(x, \lambda), & x \in(0,1],\end{cases}
$$

be the solution of (1.1) satisfying the following initial conditions:

$$
\left(\begin{array}{ll}
\mathfrak{y}_{11}(-1, \lambda) & \mathfrak{y}_{12}\left(0^{+}, \lambda\right) \\
\mathfrak{y}_{21}(-1, \lambda) & \mathfrak{y}_{22}\left(0^{+}, \lambda\right)
\end{array}\right)=\left(\begin{array}{cc}
\cos \alpha & \delta^{-1} \mathfrak{y}_{11}\left(0^{-}, \lambda\right) \\
\sin \alpha & \delta^{-1} \mathfrak{y}_{21}\left(0^{-}, \lambda\right)
\end{array}\right)
$$

In [26], Tharwat proved the existence and uniqueness of $(2.2)$. Since $\mathfrak{y}(\cdot, \lambda)$ satisfies (1.2), then the eigenvalues of problem (1.1)-(1.5) are the zeros of the function (see Lemma 2.4 of $[26, \mathrm{p} .8])$

$$
\Delta(\lambda)=\delta^{2}\left(\left(a_{1}+\lambda \sin \beta\right) \mathfrak{y}_{12}(1, \lambda)-\left(a_{2}+\lambda \cos \beta\right) \mathfrak{y}_{22}(1, \lambda)\right) .
$$

Notice that both $\mathfrak{y}(\cdot, \lambda)$ and $\Delta(\lambda)$ are entire functions of $\lambda$, and $\mathfrak{y}(\cdot, \lambda)$ satisfies the system of integral equations (cf. [26])

$$
\begin{aligned}
\mathfrak{y}_{11}(x, \lambda)= & \cos (\lambda(x+1)+\alpha)-\mathcal{S}_{-1,1} \mathfrak{y}_{11}(x, \lambda)-\widetilde{\mathcal{S}}_{-1,2} \mathfrak{y}_{21}(x, \lambda), \\
\mathfrak{y}_{21}(x, \lambda)= & \sin (\lambda(x+1)+\alpha)+\widetilde{\mathcal{S}}_{-1,1} \mathfrak{y}_{11}(x, \lambda)-\mathcal{S}_{-1,2} \mathfrak{y}_{21}(x, \lambda), \\
\mathfrak{y}_{12}(x, \lambda)= & \frac{1}{\delta} \mathfrak{y}_{11}\left(0^{-}, \lambda\right) \cos (\lambda x)-\frac{1}{\delta} \mathfrak{y}_{21}\left(0^{-}, \lambda\right) \sin (\lambda x) \\
& -\mathcal{S}_{0,1} \mathfrak{y}_{12}(x, \lambda)-\widetilde{\mathcal{S}}_{0,2} \mathfrak{y}_{22}(x, \lambda), \\
\mathfrak{y}_{22}(x, \lambda)= & \frac{1}{\delta} \mathfrak{y}_{11}\left(0^{-}, \lambda\right) \sin (\lambda x)+\frac{1}{\delta} \mathfrak{y}_{21}\left(0^{-}, \lambda\right) \cos (\lambda x) \\
& +\widetilde{\mathcal{S}}_{0,1} \mathfrak{y}_{12}(x, \lambda)-\mathcal{S}_{0,2} \mathfrak{y}_{22}(x, \lambda),
\end{aligned}
$$


where $\mathcal{S}_{-1, i}, \widetilde{\mathcal{S}}_{-1, i}, \mathcal{S}_{0, i}$ and $\widetilde{\mathcal{S}}_{0, i}, i=1,2$, are the Volterra integral operators defined by

$$
\begin{aligned}
& \mathcal{S}_{-1, i} \varphi(x, \lambda):=\int_{-1}^{x} \sin \lambda(x-t) r_{i}(t) \varphi(t, \lambda) d t, \\
& \widetilde{\mathcal{S}}_{-1, i} \varphi(x, \lambda):=\int_{-1}^{x} \cos \lambda(x-t) r_{i}(t) \varphi(t, \lambda) d t, \\
& \mathcal{S}_{0, i} \varphi(x, \lambda):=\int_{0}^{x} \sin \lambda(x-t) r_{i}(t) \varphi(t, \lambda) d t, \\
& \widetilde{\mathcal{S}}_{0, i} \varphi(x, \lambda):=\int_{0}^{x} \cos \lambda(x-t) r_{i}(t) \varphi(t, \lambda) d t .
\end{aligned}
$$

For convenience, we define the constants

$$
\begin{aligned}
& c_{1}:=\int_{-1}^{0}\left[\left|r_{1}(t)\right|+\left|r_{2}(t)\right|\right] d t, \quad c_{2}:=c_{1} \exp \left(c_{1}\right), \\
& c_{3}:=\int_{0}^{1}\left[\left|r_{1}(t)\right|+\left|r_{2}(t)\right|\right] d t, \quad c_{4}:=c_{2}+\frac{2}{|\delta|}\left(1+c_{2}\right), \\
& c_{5}:=\max \left\{\left|a_{1}\right|+\left|a_{2}\right|,|\sin \beta|+|\cos \beta|\right\} .
\end{aligned}
$$

Define $\mathfrak{z}_{-1, i}(\cdot, \lambda)$ and $\mathfrak{z}_{0, i}(\cdot, \lambda), i=1,2$, to be

$$
\begin{aligned}
& \mathfrak{z}_{-1,1}(x, \lambda):=\mathcal{S}_{-1,1} \mathfrak{y}_{11}(x, \lambda)+\widetilde{\mathcal{S}}_{-1,2} \mathfrak{y}_{21}(x, \lambda), \\
& \mathfrak{z}_{-1,2}(x, \lambda):=\widetilde{\mathcal{S}}_{-1,1} \mathfrak{y}_{11}(x, \lambda)-\mathcal{S}_{-1,2} \mathfrak{y}_{21}(x, \lambda), \\
& \mathfrak{z}_{0,1}(x, \lambda):=\mathcal{S}_{0,1} \mathfrak{y}_{12}(x, \lambda)+\widetilde{\mathcal{S}}_{0,2} \mathfrak{y}_{22}(x, \lambda), \\
& \mathfrak{z}_{0,2}(x, \lambda):=\widetilde{\mathcal{S}}_{0,1} \mathfrak{y}_{12}(x, \lambda)-\mathcal{S}_{0,2} \mathfrak{y}_{22}(x, \lambda) .
\end{aligned}
$$

Lemma 2.1 The functions $\mathfrak{z}_{-1,1}(x, \lambda)$ and $\mathfrak{z}_{-1,2}(x, \lambda)$ are entire in $\lambda$ for any fixed $x \in[-1,0)$ and satisfy the growth condition

$$
\left|\mathfrak{z}_{-1,1}(x, \lambda)\right|,\left|\mathfrak{z}_{-1,2}(x, \lambda)\right| \leq 2 c_{2} e^{|\Im \lambda|(x+1)}, \quad \lambda \in \mathbb{C}
$$

Proof Since $\mathfrak{z}_{-1,1}(x, \lambda)=\mathcal{S}_{-1,1} \mathfrak{y}_{11}(x, \lambda)+\widetilde{\mathcal{S}}_{-1,2} \mathfrak{y}_{21}(x, \lambda)$, then from (2.4) and (2.5) we obtain

$$
\begin{aligned}
\mathfrak{z}_{-1,1}(x, \lambda)= & \mathcal{S}_{-1,1} \cos (\lambda(x+1)+\alpha)+\widetilde{\mathcal{S}}_{-1,2} \sin (\lambda(x+1)+\alpha) \\
& -\mathcal{S}_{-1,1} \mathfrak{z}-1,1 \\
& (x, \lambda)+\widetilde{\mathcal{S}}_{-1,2} \mathfrak{z}_{-1,2}(x, \lambda) .
\end{aligned}
$$

Using the inequalities $|\sin z| \leq e^{|\Im z|}$ and $|\cos z| \leq e^{|\Im z|}$ for $z \in \mathbb{C}$ leads for $\lambda \in \mathbb{C}$ to

$$
\begin{aligned}
\left|\mathfrak{z}_{-1,1}(x, \lambda)\right| \leq & \left|\mathcal{S}_{-1,1} \cos (\lambda(x+1)+\alpha)\right|+\left|\widetilde{\mathcal{S}}_{-1,2} \sin (\lambda(x+1)+\alpha)\right| \\
& +\left|\mathcal{S}_{-1,1} \hat{\mathfrak{z}}_{-1,1}(x, \lambda)\right|+\left|\widetilde{\mathcal{S}}_{-1,2} \mathfrak{z}_{-1,2}(x, \lambda)\right| \\
\leq & e^{|\Im \lambda|(x+1)} \int_{-1}^{x}\left[\left|r_{1}(t)\right|\left|\mathfrak{z}_{-1,1}(t, \lambda)\right|+\left|r_{2}(t)\right|\left|\mathfrak{z}_{-1,2}(t, \lambda)\right|\right] e^{-|\Im \lambda|(t+1)} d t \\
& +2 e^{|\Im \lambda|(x+1)} \int_{-1}^{x}\left[\left|r_{1}(t)\right|+\left|r_{2}(t)\right|\right] d t
\end{aligned}
$$




$$
\begin{aligned}
\leq & 2 c_{1} e^{|\Im \lambda|(x+1)} \\
& +e^{|\Im \lambda|(x+1)} \int_{-1}^{x}\left[\left|r_{1}(t)\right|\left|\mathfrak{z}_{-1,1}(t, \lambda)\right|+\left|r_{2}(t)\right|\left|\mathfrak{z}_{-1,2}(t, \lambda)\right|\right] e^{-|\Im \lambda|(t+1)} d t .
\end{aligned}
$$

The above inequality can be reduced to

$$
\begin{aligned}
& e^{-|\Im \lambda|(x+1)}\left|\mathfrak{z}_{-1,1}(x, \lambda)\right| \\
& \quad \leq 2 c_{1}+\int_{-1}^{x}\left[\left|r_{1}(t)\right|\left|\mathfrak{z}_{-1,1}(t, \lambda)\right|+\left|r_{2}(t)\right|\left|\mathfrak{z}_{-1,2}(t, \lambda)\right|\right] e^{-|\Im \lambda|(t+1)} d t .
\end{aligned}
$$

Similarly, we can prove that

$$
\begin{aligned}
& e^{-|\Im \lambda|(x+1)}\left|\mathfrak{z}_{-1,2}(x, \lambda)\right| \\
& \quad \leq 2 c_{1}+\int_{-1}^{x}\left[\left|r_{1}(t)\right|\left|\mathfrak{z}_{-1,1}(t, \lambda)\right|+\left|r_{2}(t)\right|\left|\mathfrak{z}_{-1,2}(t, \lambda)\right|\right] e^{-|\Im \lambda|(t+1)} d t
\end{aligned}
$$

Then from (2.12), (2.13) and Lemma 3.1 of [28, p.204], we obtain (2.11).

In a similar manner, we will prove the following lemma for $\mathfrak{z}_{0,1}(\cdot, \lambda)$ and $\mathfrak{z}_{0,2}(\cdot, \lambda)$.

Lemma 2.2 The functions $\mathfrak{z}_{0,1}(x, \lambda)$ and $\mathfrak{z}_{0,2}(x, \lambda)$ are entire in $\lambda$ for any fixed $x \in(0,1]$ and satisfy the growth condition

$$
\left|\mathfrak{z}_{0,1}(x, \lambda)\right|,\left|\mathfrak{z}_{0,2}(x, \lambda)\right| \leq 2 c_{3} c_{4} e^{|\Im \lambda|(x+1)}, \quad \lambda \in \mathbb{C} .
$$

Proof Since $\mathfrak{z}_{0,1}(x, \lambda)=\mathcal{S}_{0,1} \mathfrak{y}_{12}(x, \lambda)+\widetilde{\mathcal{S}}_{0,2} \mathfrak{y}_{22}(x, \lambda)$, then from (2.6) and (2.7) we obtain

$$
\begin{aligned}
& \mathfrak{z}_{0,1}(x, \lambda)=\frac{1}{\delta} \mathfrak{y}_{11}\left(0^{-}, \lambda\right) \mathcal{S}_{0,1} \cos (\lambda x)-\frac{1}{\delta} \mathfrak{y}_{21}\left(0^{-}, \lambda\right) \mathcal{S}_{0,1} \sin (\lambda x)-\mathcal{S}_{0,1 \mathfrak{z}-1,2}(x, \lambda) \\
& +\frac{1}{\delta} \mathfrak{y}_{11}\left(0^{-}, \lambda\right) \widetilde{\mathcal{S}}_{0,2} \sin (\lambda x)+\frac{1}{\delta} \mathfrak{y}_{21}\left(0^{-}, \lambda\right) \widetilde{\mathcal{S}}_{0,2} \cos (\lambda x)+\widetilde{\mathcal{S}}_{0,2} \mathfrak{z}-1,2(x, \lambda) .
\end{aligned}
$$

Then from (2.4) and (2.5) and Lemma 2.1, we get

$$
\begin{aligned}
\left|\mathfrak{z}_{0,1}(x, \lambda)\right| \leq & \frac{1}{|\delta|}\left|\mathfrak{y}_{11}\left(0^{-}, \lambda\right)\right|\left|\mathcal{S}_{0,1} \cos (\lambda x)\right|+\frac{1}{|\delta|}\left|\mathfrak{y}_{21}\left(0^{-}, \lambda\right)\right|\left|\mathcal{S}_{0,1} \sin (\lambda x)\right| \\
& +\left|\mathcal{S}_{0,1 \mathfrak{z}-1,2}(x, \lambda)\right|+\frac{1}{|\delta|}\left|\mathfrak{y}_{11}\left(0^{-}, \lambda\right)\right|\left|\widetilde{\mathcal{S}}_{0,2} \sin (\lambda x)\right| \\
& +\frac{1}{|\delta|}\left|\mathfrak{y}_{21}\left(0^{-}, \lambda\right)\right|\left|\widetilde{\mathcal{S}}_{0,2} \cos (\lambda x)\right|+\mid \widetilde{\mathcal{S}}_{0,2} \mathfrak{z}-1,2 \\
\leq & 2\left(c_{2}+\frac{2}{|\delta|}\left(1+c_{2}\right)\right) c_{3} e^{|\Im \lambda|(x+1)} \\
= & 2 c_{3} c_{4} e^{|\Im \lambda|(x+1)} .
\end{aligned}
$$

Similarly, we can prove that

$$
\left|\mathfrak{z}_{0,2}(x, \lambda)\right| \leq 2 c_{3} c_{4} e^{|\Im \lambda|(x+1)} .
$$




\section{The numerical scheme}

In this section we derive the method of computing eigenvalues of problem (1.1)-(1.5) numerically. The basic idea of the scheme is to split $\Delta(\lambda)$ into two parts a known part $\mathcal{K}(\lambda)$ and an unknown one $\mathcal{U}(\lambda)$. Then we approximate $\mathcal{U}(\lambda)$ using (1.11) to get the approximate $\Delta(\lambda)$ and then compute the approximate zeros. We first split $\Delta(\lambda)$ into two parts as follows:

$$
\Delta(\lambda):=\mathcal{K}(\lambda)+\mathcal{U}(\lambda)
$$

where $\mathcal{U}(\lambda)$ is the unknown part involving integral operators

$$
\begin{aligned}
\mathcal{U}(\lambda):= & \delta\left[a_{2} \sin \lambda-a_{1} \cos \lambda+\lambda \sin (\lambda-\beta)\right] \mathfrak{z}_{-1,1}\left(0^{-}, \lambda\right) \\
& -\delta\left[a_{1} \sin \lambda+a_{2} \cos \lambda+\lambda \cos (\lambda-\beta)\right] \mathfrak{z}_{-1,2}\left(0^{-}, \lambda\right) \\
& +\delta^{2}\left[-\left(a_{1}+\lambda \sin \beta\right) \mathfrak{z}_{0,1}(1, \lambda)+\left(a_{2}+\lambda \cos \beta\right) \mathfrak{z}_{0,2}(1, \lambda)\right]
\end{aligned}
$$

and $\mathcal{K}(\lambda)$ is the known part

$$
\mathcal{K}(\lambda):=\delta\left[a_{1} \cos (2 \lambda+\alpha)-a_{2} \sin (2 \lambda+\alpha)-\lambda \sin (2 \lambda+\alpha-\beta)\right] .
$$

Then, from Lemma 2.1 and Lemma 2.2, we have the following result.

Lemma 3.1 The function $\mathcal{U}(\lambda)$ is entire in $\lambda$ and the following estimate holds:

$$
|\mathcal{U}(\lambda)| \leq \phi(\lambda) e^{2|\Im \lambda|}
$$

where

$$
\phi(\lambda)=: M(1+|\lambda|), \quad M:=2|\delta| c_{5}\left(c_{2}+|\delta| c_{3} c_{4}\right) .
$$

Proof From (3.2) we have

$$
\begin{aligned}
|\mathcal{U}(\lambda)| \leq & |\delta|\left[\left|a_{2}\right||\sin \lambda|+\left|a_{1}\right||\cos \lambda|+|\lambda||\sin (\lambda-\beta)|\right]\left|\mathfrak{z}_{-1,1}\left(0^{-}, \lambda\right)\right| \\
& +|\delta|\left[\left|a_{1}\right||\sin \lambda|+\left|a_{2}\right||\cos \lambda|+|\lambda||\cos (\lambda-\beta)|\right]||_{-1,2}\left(0^{-}, \lambda\right) \mid \\
& +\delta^{2}\left[\left(\left|a_{1}\right|+|\lambda||\sin \beta|\right)||_{\mathfrak{z}_{0,1}}(1, \lambda)\left|+\left(\left|a_{2}\right|+|\lambda||\cos \beta|\right)\right| \mathfrak{z}_{0,2}(1, \lambda) \mid\right] .
\end{aligned}
$$

Using the inequalities $|\sin \lambda| \leq e^{|\mathfrak{\xi} \lambda|}$ and $|\cos \lambda| \leq e^{|\mathfrak{\xi} \lambda|}$ for $\lambda \in \mathbb{C}$, Lemma 2.1 and Lemma 2.2 imply (3.4).

Thus $\mathcal{U}(\lambda)$ is an entire function of exponential type $\sigma=2$. In the following we let $\lambda \in \mathbb{R}$ since all eigenvalues are real. Now we approximate the function $\mathcal{U}(\lambda)$ using the operator (1.11) where $h \in(0, \pi / 2)$ and $\omega:=(\pi-2 h) / 2$ and then, from (1.13), we obtain

$$
\left|\mathcal{U}(\lambda)-\left(\mathcal{G}_{h, N} \mathcal{U}\right)(\lambda)\right| \leq T_{h, N}(\lambda)
$$

where

$$
T_{h, N}(\lambda):=2\left|\sin \left(h^{-1} \pi \lambda\right)\right| \phi(|\Re \lambda|+h(N+1)) \frac{e^{-\omega N}}{\sqrt{\pi \omega N}} \beta_{N}(0), \quad \lambda \in \mathbb{R} .
$$


The samples $\mathcal{U}(n h)=\Delta(n h)-\mathcal{K}(n h), n \in \mathbb{Z}_{N}(\lambda)$ cannot be computed explicitly in the general case. We approximate these samples numerically by solving the initial value problems defined by (1.1) and (2.2) to obtain the approximate values $\tilde{\mathcal{U}}(n h), n \in \mathbb{Z}_{N}(\lambda)$, i.e., $\tilde{\mathcal{U}}(n h)=\widetilde{\Delta}(n h)-\mathcal{K}(n h)$. Here we use the computer algebra system MATHEMATICA to obtain approximate solutions with the required accuracy. However, a separate study for the effect of different numerical schemes and the computational costs would be interesting. Accordingly, we have the explicit expansion

$$
\left(\mathcal{G}_{h, N} \tilde{\mathcal{U}}\right)(\lambda):=\sum_{n \in \mathbb{Z}_{N}(\lambda)} \tilde{\mathcal{U}}(n h) \operatorname{sinc}\left(h^{-1} \pi \lambda-n \pi\right) G\left(\frac{\sqrt{\omega}(\lambda-n h)}{\sqrt{N} h}\right) .
$$

Therefore we get (cf. (1.16))

$$
\left|\left(\mathcal{G}_{h, N} \mathcal{U}\right)(\lambda)-\left(\mathcal{G}_{h, N} \tilde{\mathcal{U}}\right)(\lambda)\right| \leq A_{\varepsilon, N}(0), \quad \lambda \in \mathbb{R}
$$

Now let $\widetilde{\Delta}_{N}(\lambda):=\mathcal{K}(\lambda)+\left(\mathcal{G}_{h, N} \widetilde{\mathcal{U}}\right)(\lambda)$. From (3.6) and (3.9) we obtain

$$
\left|\Delta(\lambda)-\widetilde{\Delta}_{N}(\lambda)\right| \leq T_{h, N}(\lambda)+A_{\varepsilon, N}(0), \quad \lambda \in \mathbb{R} .
$$

Let $\lambda^{*}$ be an eigenvalue and $\lambda_{N}$ be its desired approximation, i.e., $\Delta\left(\lambda^{*}\right)=0$ and $\widetilde{\Delta}_{N}\left(\lambda_{N}\right)=0$. From (3.10) we have $\left|\widetilde{\Delta}_{N}\left(\lambda^{*}\right)\right| \leq T_{h, N}\left(\lambda^{*}\right)+A_{\varepsilon, N}(0)$. Define the curves

$$
a_{ \pm}(\lambda)=\widetilde{\Delta}_{N}(\lambda) \pm T_{h, N}(\lambda)+A_{\varepsilon, N}(0) .
$$

The curves $a_{+}(\lambda), a_{-}(\lambda)$ enclose the curve of $\Delta(\lambda)$ for suitably large $N$. Hence the closure interval is determined by solving $a_{ \pm}(\lambda)=0$, which gives an interval

$$
I_{\varepsilon, N}:=\left[a_{-}, a_{+}\right]
$$

It is worthwhile to mention that the simplicity of the eigenvalues guarantees the existence of approximate eigenvalues, i.e., the $\lambda_{N}$ for which $\widetilde{\Delta}_{N}\left(\lambda_{N}\right)=0$. Next we estimate the error $\left|\lambda^{*}-\lambda_{N}\right|$ for the eigenvalue $\lambda^{*}$.

Theorem 3.2 Let $\lambda^{*}$ be an eigenvalue of (1.1)-(1.5) and let $\lambda_{N}$ be its approximation. Then, for $\lambda \in \mathbb{R}$, we have the following estimate:

$$
\left|\lambda^{*}-\lambda_{N}\right|<\frac{T_{h, N}\left(\lambda_{N}\right)+A_{\varepsilon, N}(0)}{\inf _{\zeta \in I_{\varepsilon, N}}\left|\Delta^{\prime}(\zeta)\right|}
$$

where the interval $I_{\varepsilon, N}$ is defined above.

Proof Replacing $\lambda$ by $\lambda_{N}$ in (3.10), we obtain

$$
\left|\Delta\left(\lambda_{N}\right)-\Delta\left(\lambda^{*}\right)\right|<T_{h, N}\left(\lambda_{N}\right)+A_{\varepsilon, N}(0)
$$

where we have used $\widetilde{\Delta}_{N}\left(\lambda_{N}\right)=\Delta\left(\lambda^{*}\right)=0$. Using the mean value theorem yields that for some $\zeta \in J_{\varepsilon, N}:=\left[\min \left(\lambda^{*}, \lambda_{N}\right), \max \left(\lambda^{*}, \lambda_{N}\right)\right]$,

$$
\left|\left(\lambda^{*}-\lambda_{N}\right) \Delta^{\prime}(\zeta)\right| \leq T_{h, N}\left(\lambda_{N}\right)+A_{\varepsilon, N}(0), \quad \zeta \in J_{\varepsilon, N} \subset I_{\varepsilon, N} .
$$


Since $\lambda^{*}$ is simple and $N$ is sufficiently large, then $\inf _{\zeta \in I_{\varepsilon, N}}\left|\Delta^{\prime}(\zeta)\right|>0$ and we get (3.12).

\section{Numerical examples}

This section includes two examples illustrating the sinc-Gaussian method. It is clearly seen that the sinc-Gaussian method gives remarkably better results. We indicate in these two examples the effect of the amplitude error in the method by determining enclosure intervals for different values of $\varepsilon$. We also indicate the effect of $N$ and $h$ by several choices. We would like to mention that MATHEMATica has been used to obtain the exact values for these examples where eigenvalues cannot be computed concretely. MATHEMATICA is also used in rounding off the exact eigenvalues, which are square roots. Each example is presented via figures that accurately illustrate the procedure near some of the approximated eigenvalues. More explanations are given below.

Example 4.1 Consider the system

$$
\begin{aligned}
& y_{2}^{\prime}(x)-r(x) y_{1}(x)=\lambda y_{1}(x), \quad y_{1}^{\prime}(x)+r(x) y_{2}(x)=-\lambda y_{2}(x), \quad x \in[-1,0) \cup(0,1], \\
& y_{1}(-1)=0, \quad(1+\lambda) y_{1}(1)+y_{2}(1)=0, \\
& y_{1}\left(0^{-}\right)-2 y_{1}\left(0^{+}\right)=0, \quad y_{2}\left(0^{-}\right)-2 y_{2}\left(0^{+}\right)=0 .
\end{aligned}
$$

Here

$$
r_{1}(x)=r_{2}(x)=r(x)= \begin{cases}x, & x \in[-1,0), \\ x^{2}, & (0,1]\end{cases}
$$

$\alpha=\beta=\frac{\pi}{2}, a_{1}=1, a_{2}=-1$ and $\delta=2$. Direct calculations give

$$
\mathcal{K}(\lambda)=2(\cos [2 \lambda]-(1+\lambda) \sin [2 \lambda])
$$

and

$$
\Delta(\lambda)=2\left(\cos \left[\frac{1}{6}-2 \lambda\right]+(1+\lambda) \sin \left[\frac{1}{6}-2 \lambda\right]\right) .
$$

As is clearly seen, the eigenvalues cannot be computed explicitly. The following three ta-

\begin{tabular}{|c|c|c|c|c|c|}
\hline$\lambda_{k}$ & & $\lambda_{-2}$ & $\lambda_{-1}$ & $\lambda_{0}$ & $\lambda_{1}$ \\
\hline Exact $\lambda_{k}$ & & -1.9050594725435388 & -0.8005149927957496 & 0.3944055848645847 & 1.8242788740449205 \\
\hline$\lambda_{k, N} \quad h=0.8$ & $N=10$ & -1.9050945328700728 & -0.8005149844410676 & 0.3943794190610962 & 1.8242617833701285 \\
\hline$\omega=0.7714$ & $N=20$ & -1.9050594925575182 & -0.8005149927903844 & 0.39440557044475477 & 1.8242788645444055 \\
\hline$h=0.2$ & $N=10$ & -1.9050594937724747 & -0.8005149927866473 & 0.3944055855507727 & 1.8242788693330168 \\
\hline$\omega=1.3714$ & $N=20$ & -1.905059472543563 & -0.8005149927957529 & 0.39440558486458616 & 1.824278874044914 \\
\hline
\end{tabular}
bles (Tables 1,2,3) indicate the application of our technique to this problem and the effect of $\varepsilon$. By exact we mean the zeros of $\Delta(\lambda)$ computed by Mathematica.

Figures 1 and 2 illustrate the enclosure intervals dominating $\lambda_{-2}$ for $N=20, h=0.2$ and $\varepsilon=10^{-2}, \varepsilon=10^{-5}$, respectively. The middle curve represents $\Delta(\lambda)$, while the upper and

Table 1 The approximation $\lambda_{k, N}$ and the exact solution $\lambda_{k}$ for different choices of $h$ and $N$ 
Table 2 Absolute error $\left|\lambda_{k}-\lambda_{k, N}\right|$

\begin{tabular}{llllll}
\hline $\boldsymbol{\lambda}_{\boldsymbol{k}}$ & & $\boldsymbol{\lambda}_{\mathbf{- 2}}$ & $\boldsymbol{\lambda}_{\mathbf{- 1}}$ & $\boldsymbol{\lambda}_{\mathbf{0}}$ & $\boldsymbol{\lambda}_{\mathbf{1}}$ \\
\hline$h=0.8$ & $N=10$ & $3.50603 \times 10^{-5}$ & $8.35468 \times 10^{-9}$ & $2.61658 \times 10^{-5}$ & $1.70907 \times 10^{-5}$ \\
& $N=20$ & $2.0014 \times 10^{-8}$ & $5.36515 \times 10^{-12}$ & $1.44198 \times 10^{-8}$ & $9.50052 \times 10^{-9}$ \\
$h=0.2$ & $N=10$ & $2.12289 \times 10^{-8}$ & $9.10227 \times 10^{-12}$ & $6.86188 \times 10^{-10}$ & $4.7119 \times 10^{-9}$ \\
& $N=20$ & $2.42029 \times 10^{-14}$ & $3.33067 \times 10^{-15}$ & $3.33067 \times 10^{-15}$ & $6.43929 \times 10^{-15}$
\end{tabular}

Table 3 For $N=20$ and $h=0.2$, the exact solutions $\lambda_{k}$ are all inside the interval $\left[a_{-}, a_{+}\right]$for different values of $\varepsilon$

\begin{tabular}{lllll}
\hline $\boldsymbol{\lambda}_{\boldsymbol{k}}$ & $\boldsymbol{\lambda}_{\mathbf{- 2}}$ & $\boldsymbol{\lambda}_{\mathbf{- 1}}$ & $\boldsymbol{\lambda}_{\mathbf{0}}$ & $\boldsymbol{\lambda}_{\mathbf{1}}$ \\
\hline Exact $\boldsymbol{\lambda}_{k}$ & -1.9050594725435388 & -0.8005149927957496 & 0.3944055848645847 & 1.8242788740449205 \\
$I_{\varepsilon, N}, \varepsilon=10^{-2}$ & {$[-1.9270913,-1.8826416]$} & {$[-0.8259079,-0.7752883]$} & {$[0.3752245,0.4132930]$} & {$[1.8121593,1.8363114]$} \\
$I_{\varepsilon, N}, \varepsilon=10^{-5}$ & {$[-1.9050816,-1.9050372]$} & {$[-0.8005402,-0.8004897]$} & {$[0.3944246,0.3944055]$} & {$[1.8242667,1.8242909]$} \\
\hline
\end{tabular}

Figure 1 The enclosure interval dominating $\lambda_{-2}$ for $h=0.2, N=20$ and $\varepsilon=10^{-2}$.

Figure 2 The enclosure interval
for $h=0.2, N=20$ and $\varepsilon=10^{-5}$.

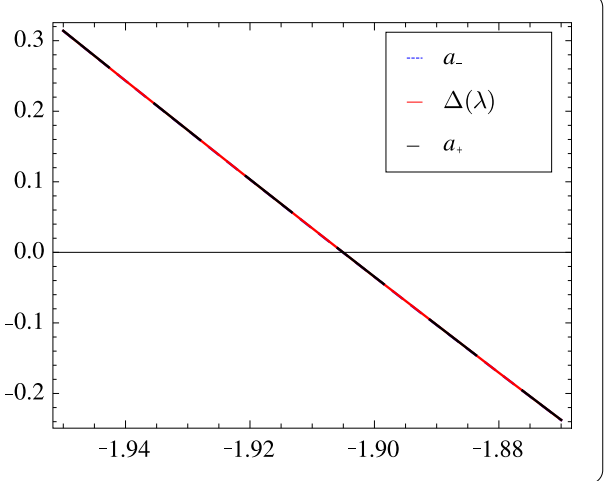

lower curves represent the curves of $a_{+}(\lambda), a_{-}(\lambda)$, respectively. We notice that when $\varepsilon=$ $10^{-5}$, the two curves are almost identical. Similarly, Figures 3 and 4 illustrate the enclosure intervals dominating $\lambda_{-1}$ for $h=0.2, N=20$ and $\varepsilon=10^{-2}, \varepsilon=10^{-5}$, respectively.

Example 4.2 In this example we consider the system

$$
\begin{aligned}
& y_{2}^{\prime}(x)-r(x) y_{1}(x)=\lambda y_{1}(x), \quad y_{1}^{\prime}(x)+r(x) y_{2}(x)=-\lambda y_{2}(x), \quad x \in[-1,0) \cup(0,1], \\
& \sqrt{3} y_{1}(-1)-y_{2}(-1)=0, \quad\left(1+\frac{1}{2} \lambda\right) y_{1}(1)-\left(1+\frac{\sqrt{3}}{2} \lambda\right) y_{2}(1)=0, \\
& y_{1}\left(0^{-}\right)-3 y_{1}\left(0^{+}\right)=0, \quad y_{2}\left(0^{-}\right)-3 y_{2}\left(0^{+}\right)=0,
\end{aligned}
$$




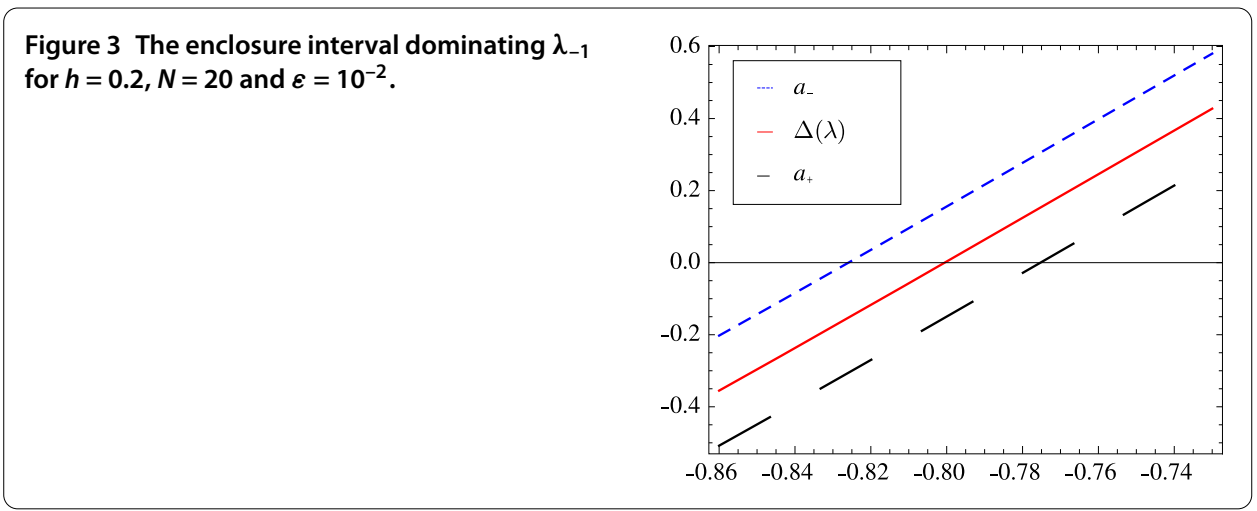

Figure 4 The enclosure interval dominating $\lambda_{-1}$ for $h=0.2, N=20$ and $\varepsilon=10^{-5}$.

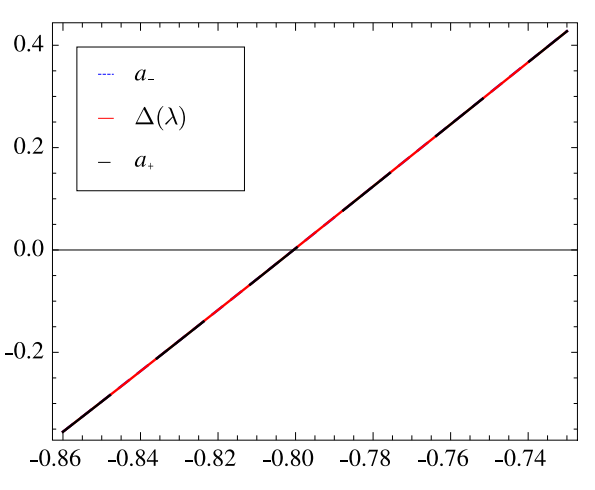

Table 4 The approximation $\lambda_{k, N}$ and the exact solution $\lambda_{k}$ for different choices of $h$ and $N$

\begin{tabular}{|c|c|c|c|c|c|c|}
\hline \multicolumn{3}{|l|}{$\lambda_{k}$} & \multirow{2}{*}{$\begin{array}{l}\boldsymbol{\lambda}_{\mathbf{- 2}} \\
-1.443241990338957\end{array}$} & \multirow{2}{*}{$\frac{\lambda_{-1}}{-0.5507950329405884}$} & \multirow{2}{*}{$\begin{array}{l}\lambda_{0} \\
0.8894376317278696\end{array}$} & \multirow{2}{*}{$\frac{\lambda_{1}}{2.427882996831557}$} \\
\hline Exac & $t \lambda_{k}$ & & & & & \\
\hline \multirow{4}{*}{$\lambda_{k, N}$} & $h=0.6$ & $N=10$ & -1.4432116741528003 & -0.5507870771754422 & 0.8894392796056301 & 2.4278845029050586 \\
\hline & $\omega=0.9714$ & $N=20$ & -1.4432419877352867 & -0.5507950322921764 & 0.8894376316344037 & 2.427882996941257 \\
\hline & $h=0.1$ & $N=10$ & -1.4432 & -0.5507950143369327 & 0.8894376262695777 & 30194325765 \\
\hline & $\omega=1.4714$ & $N=20$ & -1.4432419903389377 & -0.5507950329405837 & 0.88943763172786576 & 2.4278829968315647 \\
\hline
\end{tabular}

where

$$
r_{1}(x)=r_{2}(x)=r(x)= \begin{cases}x+1, & x \in[-1,0), \\ x, & (0,1],\end{cases}
$$

$a_{1}=a_{2}=1, \alpha=\frac{\pi}{3}, \beta=\frac{\pi}{6}$ and $\delta=3$. Direct calculations give

$$
\mathcal{K}(\lambda)=3\left[\cos \left[\frac{\pi}{3}+2 \lambda\right]-\lambda \sin \left[\frac{\pi}{6}+2 \lambda\right]-\sin \left[\frac{\pi}{3}+2 \lambda\right]\right]
$$

and

$$
\Delta(\lambda)=-\frac{3}{2}[(-1+\sqrt{3}+\lambda) \cos [1+2 \lambda]+(1+\sqrt{3}+\sqrt{3} \lambda) \sin [1+2 \lambda]] .
$$

Tables 4,5 , give the exact eigenvalues $\left\{\lambda_{k}\right\}_{k=-2}^{1}$ and their approximate ones for different values of $h, N, \varepsilon$. In Table 6, we give the absolute error for different values of $h$ and $N$.

Here Figures 5, 6, 7, 8 illustrate the enclosure intervals dominating $\lambda_{0}$ and $\lambda_{1}$ for $h=0.1$, $N=20$ and $\varepsilon=10^{-2}, \varepsilon=10^{-5}$, respectively. 
Table 5 For $N=20$ and $h=0.1$, the exact solutions $\lambda_{k}$ are all inside the interval $\left[a_{-}, a_{+}\right]$for different values of $\varepsilon$

\begin{tabular}{lllll}
\hline $\boldsymbol{\lambda}_{\boldsymbol{k}}$ & $\boldsymbol{\lambda}_{\mathbf{- 2}}$ & $\boldsymbol{\lambda}_{\mathbf{- 1}}$ & $\boldsymbol{\lambda}_{\mathbf{0}}$ & $\boldsymbol{\lambda}_{\mathbf{1}}$ \\
\hline Exact $\boldsymbol{\lambda}_{k}$ & -1.443241990338957 & -0.5507950329405884 & 0.88943763172786966 & 2.427882996831557 \\
$I_{\varepsilon, N} \varepsilon=10^{-2}$ & {$[-1.4716489,-1.4144426]$} & {$[-0.5736938,-0.5287366]$} & {$[0.8789632,0.8998212]$} & {$[2.4214822,2.4342626]$} \\
$I_{\varepsilon, N} \varepsilon=10^{-5}$ & {$[-1.4432705,-1.4432134]$} & {$[-0.5508174,-0.5507725]$} & {$[0.8894272,0.8894480]$} & {$[2.4278766,2.4278893]$} \\
\hline
\end{tabular}

Table 6 Absolute error $\left|\lambda_{k}-\lambda_{k, N}\right|$

\begin{tabular}{llllll}
\hline $\boldsymbol{\lambda}_{\boldsymbol{k}}$ & & $\boldsymbol{\lambda}_{\mathbf{- 2}}$ & $\boldsymbol{\lambda}_{\mathbf{- 1}}$ & $\boldsymbol{\lambda}_{\mathbf{0}}$ & $\boldsymbol{\lambda}_{\mathbf{1}}$ \\
\hline$h=0.6$ & $N=10$ & $3.03162 \times 10^{-5}$ & $7.95577 \times 10^{-6}$ & $1.64788 \times 10^{-6}$ & $1.50607 \times 10^{-6}$ \\
& $N=20$ & $2.60367 \times 10^{-9}$ & $6.48412 \times 10^{-10}$ & $9.34659 \times 10^{-11}$ & $1.097 \times 10^{-10}$ \\
$h=0.1$ & $N=10$ & $3.60989 \times 10^{-8}$ & $1.86037 \times 10^{-8}$ & $5.45829 \times 10^{-9}$ & $2.2601 \times 10^{-8}$ \\
& $N=20$ & $1.93179 \times 10^{-14}$ & $4.66294 \times 10^{-15}$ & $3.88578 \times 10^{-15}$ & $7.54952 \times 10^{-15}$ \\
\hline
\end{tabular}

Figure 5 The enclosure interval dominating $\lambda_{0}$ for $h=0.1, N=20$ and $\varepsilon=10^{-2}$.

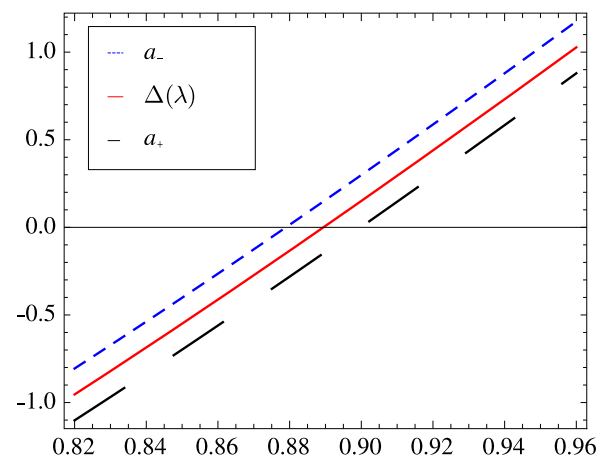

Figure 6 The enclosure interval dominating $\lambda_{0}$ for $h=0.1, N=20$ and $\varepsilon=10^{-5}$.

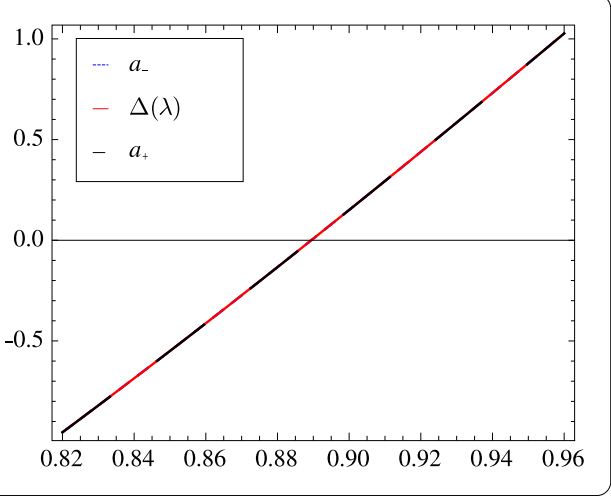

Figure 7 The enclosure interval dominating $\lambda_{1}$ for $h=0.1, N=20$ and $\varepsilon=10^{-2}$.

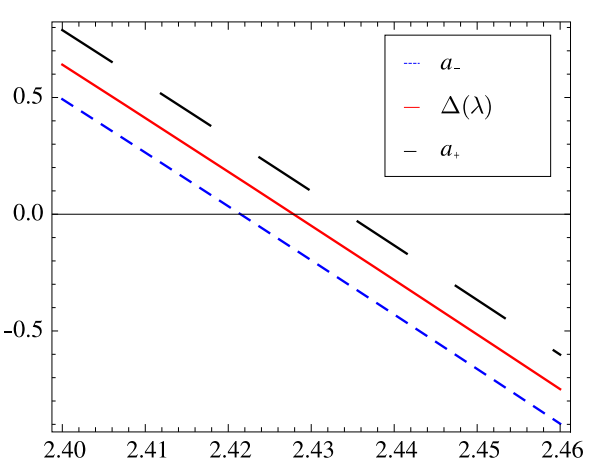




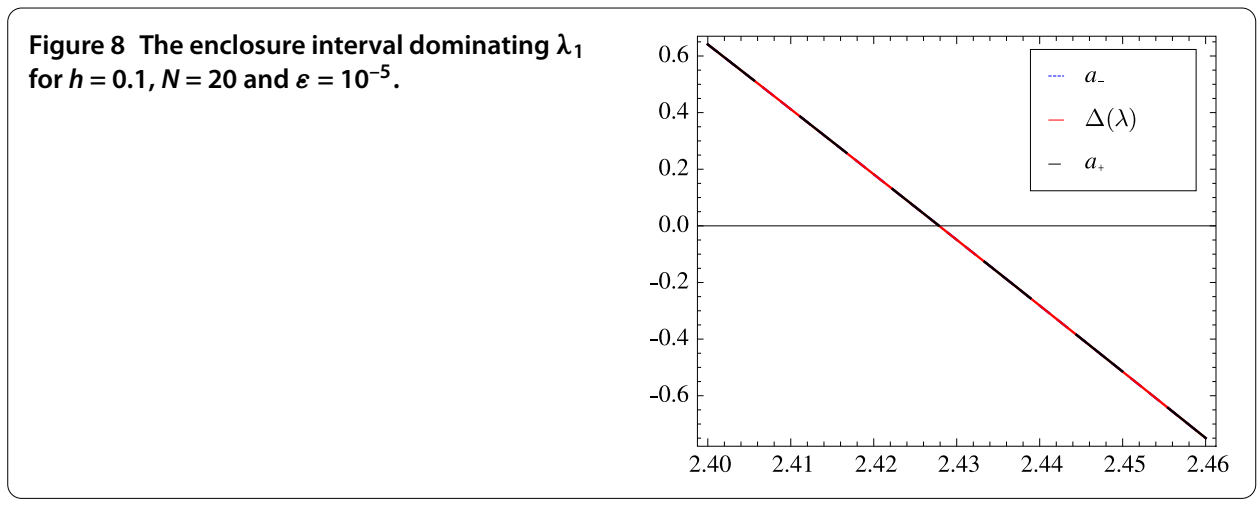

\section{Competing interests}

The authors declare that they have no competing interests.

\section{Authors' contributions}

The authors have equal contributions to each part of this article. All the authors read and approved the final manuscript.

\section{Author details}

${ }^{1}$ Department of Mathematics, Faculty of Science, King Abdulaziz University, North Jeddah, Saudi Arabia. ${ }^{2}$ Permanent address: Department of Mathematics, Faculty of Science, Beni-Suef University, Beni-Suef, Egypt. ${ }^{3}$ Department of Mathematics, University College, Umm Al-Qura University, P.O. Box 8140, Makkah, Saudi Arabia.

\section{Acknowledgements}

This research was supported by a grant from the Institute of Scientific Research at Umm Al-Qura University, Saudi Arabia.

Received: 18 December 2013 Accepted: 27 February 2014 Published: 11 Mar 2014

\section{References}

1. Kotel'nikov, V: On the carrying capacity of the 'ether' and wire in telecommunications. In: Material for the First All-Union Conference on Questions of Communications, vol. 55, pp. 55-64. Izd. Red. Upr. Svyazi RKKA, Moscow (1933) (Russian)

2. Shannon, CE: Communications in the presence of noise. Proc. IRE 37, 10-21 (1949)

3. Whittaker, ET: On the functions which are represented by the expansion of the interpolation theory. Proc. R. Soc. Edinb., Sect. A 35, 181-194 (1915)

4. Butzer, PL, Schmeisser, G, Stens, RL: An introduction to sampling analysis. In: Marvasti, F (ed.) Nonuniform Sampling: Theory and Practices, pp. 17-121. Kluwer Academic, New York (2001)

5. Kowalski, M, Sikorski, K, Stenger, F: Selected Topics in Approximation and Computation. Oxford University Press, London (1995)

6. Lund, J, Bowers, K: Sinc Methods for Quadrature and Differential Equations. SIAM, Philadelphia (1992)

7. Stenger, F: Numerical methods based on Whittaker cardinal, or sinc functions. SIAM Rev. 23, 156-224 (1981)

8. Stenger, F: Numerical Methods Based on Sinc and Analytic Functions. Springer, New York (1993)

9. Boumenir, A: Higher approximation of eigenvalues by the sampling method. BIT Numer. Math. 40, 215-225 (2000)

10. Boumenir, A: Sampling and eigenvalues of non-self-adjoint Sturm-Liouville problems. SIAM J. Sci. Comput. 23, 219-229 (2001)

11. Tharwat, MM, Bhrawy, AH, Yildirim, A: Numerical computation of eigenvalues of discontinuous Sturm-Liouville problems with parameter dependent boundary conditions using sinc method. Numer. Algorithms 63, 27-48 (2013)

12. Tharwat, MM, Bhrawy, AH, Yildirim, A: Numerical computation of eigenvalues of discontinuous Dirac system using sinc method with error analysis. Int. J. Comput. Math. 89, 2061-2080 (2012)

13. Butzer, PL, Stens, RL: A modification of the Whittaker-Kotel'nikov-Shannon sampling series. Aequ. Math. 28, 305-311 (1985)

14. Gervais, R, Rahman, Ql, Schmeisser, G: A bandlimited function simulating a duration-limited one. In: Butzer, PL, Stens, RL (eds.) Approximation Theory and Functional Analysis, pp. 355-362. Birkhäuser, Basel (1984)

15. Stens, RL: Sampling by generalized kernels. In: Higgins, JR, Stens, RL (eds.) Sampling Theory in Fourier and Signal Analysis: Advanced Topics, pp. 130-157. Oxford University Press, Oxford (1999)

16. Schmeisser, G, Stenger, F: Sinc approximation with a Gaussian multiplier. Sampl. Theory Signal Image Process. 6 199-221 (2007)

17. Qian, L: On the regularized Whittaker-Kotel'nikov-Shannon sampling formula. Proc. Am. Math. Soc. 131, 1169-1176 (2002)

18. Qian, L, Creamer, DB: A modification of the sampling series with a Gaussian multiplier. Sampl. Theory Signal Image Process. 5, 1-20 (2006)

19. Qian, L, Creamer, DB: Localized sampling in the presence of noise. Appl. Math. Lett. 19, 351-355 (2006)

20. Annaby, MH, Asharabi, RM: Computing eigenvalues of boundary-value problems using sinc-Gaussian method. Sampl. Theory Signal Image Process. 7, 293-312 (2008)

21. Tharwat, MM, Bhrawy, AH: Computation of eigenvalues of discontinuous Dirac system using Hermite interpolation technique. Adv. Differ. Equ. (2012). doi:10.1186/1687-1847-2012-59 
22. Tharwat, MM, Yildirim, A, Bhrawy, AH: Sampling of discontinuous Dirac systems. Numer. Funct. Anal. Optim. 34 323-348 (2013)

23. Bhrawy, AH, Tharwat, MM, Al-Fhaid, A: Numerical algorithms for computing eigenvalues of discontinuous Dirac system using sinc-Gaussian method. Abstr. Appl. Anal. (2012). doi:10.1155/2012/925134

24. Annaby, MH, Tharwat, MM: A sinc-Gaussian technique for computing eigenvalues of second-order linear pencils. Appl. Numer. Math. 63, 129-137 (2013)

25. Tharwat, MM, Bhrawy, AH, Alofi, AS: Approximation of eigenvalues of discontinuous Sturm-Liouville problems with eigenparameter in all boundary conditions. Bound. Value Probl. (2013). doi:10.1186/1687-2770-2013-132

26. Tharwat, MM: On sampling theories and discontinuous Dirac systems with eigenparameter in the boundary conditions. Bound. Value Probl. (2013). doi:10.1186/1687-2770-2013-65

27. Levitan, BM, Sargsjan, IS: Introduction to Spectral Theory: Self Adjoint Ordinary Differential Operators. Translation of Mathematical Monographs, vol. 39. Am. Math. Soc., Providence (1975)

28. Levitan, BM, Sargsjan, IS: Sturm-Liouville and Dirac Operators. Kluwer Academic, Dordrecht (1991)

29. Tharwat, MM: Discontinuous Sturm-Liouville problems and associated sampling theories. Abstr. Appl. Anal. (2011). doi:10.1155/2011/610232

10.1186/1687-2770-2014-51

Cite this article as: Tharwat and Al-Harbi: Approximation of eigenvalues of boundary value problems. Boundary Value Problems 2014, 2014:51

\section{Submit your manuscript to a SpringerOpen ${ }^{\odot}$ journal and benefit from:}

- Convenient online submission

Rigorous peer review

- Immediate publication on acceptance

- Open access: articles freely available online

High visibility within the field

Retaining the copyright to your article 